\title{
Adding Value to Crystallographically-Derived Ligand Knowledge Bases
}

Natalie Fey, Stephanie E. Harris, Jeremy N. Harvey and A. Guy Orpen.

School of Chemistry, University of Bristol, Cantock's Close, Bristol BS8 1TS, U.K.

\section{Supporting Information}


Table S1: Summary of crystal structure reports where unusual/outlier structural features have been discussed in detail.

\begin{tabular}{|c|c|c|c|c|}
\hline No. & CSD Refcode & Complex & Why Outlier? & Author Expla \\
\hline S1 & RIWXEA & $\gamma_{\mathrm{tBu}}$ & $\begin{array}{l}\text { Only disphenoidal geometry reported } \\
\text { for } \mathrm{Cr}(\mathrm{II}) \text { complexes }\left(\mathrm{A} 1=169^{\circ} \text {, }\right. \\
\left.\mathrm{A} 2=107^{\circ}\right) \text {. Majority of } \mathrm{Cr}(\mathrm{II}) \\
\text { complexes are square planar. }\end{array}$ & $\begin{array}{l}\text { Tris(pyrazolyl)borate }(\mathrm{Tp}) \mathrm{li}_{1} \\
\text { enforce tetrahedral geometry } \\
\text { molecular orbital arguments } \\
\text { symmetry lowering distortio] } \\
\text { high-spin } \mathrm{d}^{4} \text { tetrahedral. }\end{array}$ \\
\hline S2 & ODIHOY & & $\begin{array}{l}\text { Distorted geometry for } \mathrm{Cr}(\mathrm{II}) \\
\left(\mathrm{A} 1=150^{\circ}, \mathrm{A} 2=133^{\circ}\right) \text {, cf. S1. }\end{array}$ & $\begin{array}{l}\text { Steric bulk of ortho-phenyl s } \\
\text { distortion from square planaı }\end{array}$ \\
\hline S3 & ZAZYAA10 & $\mathrm{Ru}(\mathrm{CO})_{2}\left(\mathrm{P}^{\mathrm{t}} \mathrm{Bu}_{2} \mathrm{Me}\right)_{2}$ & $\begin{array}{l}\text { Distorted geometry for 4-coordinate } \\
\mathrm{Ru}(0) \text { complex }\left(\mathrm{A} 1=166^{\circ},\right. \\
\left.\mathrm{A} 2=133^{\circ}\right) \text {. }\end{array}$ & $\begin{array}{l}\text { Described as trigonal bipyrai } \\
\text { equatorial ligand, supported } \\
\text { and analysis of electronic str }\end{array}$ \\
\hline $\begin{array}{l}\text { S4, } \\
\text { S5 }\end{array}$ & $\begin{array}{l}\text { BAJWAL } \\
(\mathrm{X}=\mathrm{Br}), \\
\text { BAJWEP }(\mathrm{X}=\mathrm{I})\end{array}$ & & $\begin{array}{l}\text { Disphenoidal geometry for } \mathrm{Ni}(\mathrm{II})(\mathrm{A} 1 \\
\left.=158^{\circ}(4), 154^{\circ}(5), \mathrm{A} 2=116^{\circ}\right) . \\
\text { Observe }<10 \mathrm{Ni}(\mathrm{II}) \text { disphenoidal } \\
\text { geometries; main } \mathrm{Ni}(\mathrm{II}) \text { geometry } \\
\text { square planar }(>2000 \text { structures }) .\end{array}$ & $\begin{array}{l}\text { Terminal methyl substituents } \\
\text { exert steric hindrance, so bul } \\
\text { iodide distorted out of plane. }\end{array}$ \\
\hline S6 & OKISUW & But- & $\begin{array}{l}\text { Square planar geometry for } \mathrm{Ru}(\mathrm{II}) \\
\text { complex }\left(\mathrm{A} 1=178^{\circ}, \mathrm{A} 2=175^{\circ}\right) \text {. }\end{array}$ & $\begin{array}{l}\text { Tridentate PNP ligand forces } \\
\text { geometry, additional experin } \\
\text { paramagnetic complex. Tripl } \\
(\mathrm{S}=1) \text { confirmed by DFT cal }\end{array}$ \\
\hline
\end{tabular}


Table S2: Key structural parameters for DFT optimized complexes.

\begin{tabular}{|c|c|c|c|c|c|}
\hline No. & Complex & Input Description & M-L / $\AA$ & $\mathrm{A} 1, \mathrm{~A} 2 /^{\circ}$ & Oth \\
\hline 2 & $\mathrm{~W}(\mathrm{DMP})_{4}, \mathrm{DMP}=\mathrm{O}-2,6-\mathrm{Me}_{2}-\mathrm{C}_{6} \mathrm{H}_{3}$ & $\begin{array}{l}\text { From XRD } \\
\text { From tetrahedral } \\
D_{4} \text {, square planar } \\
S_{4} \text {, tetrahedral }\end{array}$ & $\begin{array}{l}(1.880,1.876) * 2 \\
(1.879,1.883) * 2 \\
1.876 * 4 \\
1.922 * 4\end{array}$ & $\begin{array}{l}178,175 \\
178,178 \\
180,180 \\
115,115\end{array}$ & $\begin{array}{l}\mathrm{C}-\mathrm{O} \\
168 \\
\mathrm{C}-\mathrm{O} \\
\mathrm{C}-\mathrm{O} \\
\mathrm{C}-\mathrm{O}\end{array}$ \\
\hline 3a & {$\left[\mathrm{V}(\mathrm{DMP})_{4}\{\mathrm{Li}(\mathrm{thf})\}_{2}\right]$} & $\begin{array}{l}\text { From simplified XRD, } \\
\mathrm{S}=3 / 2\end{array}$ & $\begin{array}{l}\mathrm{V}-\mathrm{O}=2.007,2.095,2.011 \\
2.088 ; \mathrm{V}-\mathrm{Li}=2.823,2.822\end{array}$ & 164,150 & $\begin{array}{l}\mathrm{C}-\mathrm{O} \\
135\end{array}$ \\
\hline 4 & $\mathrm{~V}\left(\mathrm{O}-2,6-{ }^{\mathrm{t}} \mathrm{Bu}_{2}-4-\mathrm{Me}-\mathrm{C}_{6} \mathrm{H}_{2}\right)_{2} \mathrm{py}_{2}$ & From $\mathrm{XRD}, \mathrm{S}=3 / 2$ & $\begin{array}{l}\mathrm{V}-\mathrm{O}=1.930 * 2 ; \mathrm{V}-\mathrm{N}= \\
2.305\end{array}$ & 180,180 & $\mathrm{C}-\mathrm{O}$ \\
\hline $5 \mathbf{a}$ & $\operatorname{Re}(\mathrm{DMP})_{4}$ & $\begin{array}{l}\text { From simplified XRD } \\
\text { Tetrahedral geometry }\end{array}$ & $\begin{array}{l}1.933,1.840,1.931,1.844 \\
(1.877,1.884) * 2\end{array}$ & $\begin{array}{l}175,174 \\
179,179\end{array}$ & $\begin{array}{l}\mathrm{C}-\mathrm{O} \\
145 \\
\mathrm{C}-\mathrm{O}\end{array}$ \\
\hline 6 & $\mathrm{~V}\left(\mathrm{O}-2,6-\mathrm{Ph}_{2}-\mathrm{C}_{6} \mathrm{H}_{3}\right)_{2}($ tmeda $)$ & From $\mathrm{XRD}, \mathrm{S}=3 / 2$ & $\begin{array}{l}\mathrm{V}-\mathrm{O}=2.028,2.036 ; \mathrm{V}-\mathrm{N} \\
=2.314,2.276\end{array}$ & 170,82 & $\mathrm{M}-\mathrm{C}$ \\
\hline 6a & $\mathrm{V}\left(\mathrm{O}-2-\mathrm{Ph}-6-\mathrm{Me}-\mathrm{C}_{6} \mathrm{H}_{3}\right)_{2}$ (tmeda) & simplified $\mathbf{6}, \mathrm{S}=3 / 2$ & $\begin{array}{l}\mathrm{V}-\mathrm{O}=1.921,1.942 ; \mathrm{V}-\mathrm{N} \\
=2.280,2.301\end{array}$ & 118,80 & $\mathrm{M}-\mathrm{C}$ \\
\hline 7 & $\mathrm{RuCl}_{2}\left(\mathrm{PPh}_{2}\left(2,6-\mathrm{Me}_{2}-\mathrm{C}_{6} \mathrm{H}_{3}\right)_{2}\right.$ & From XRD & $\begin{array}{l}\mathrm{Ru}-\mathrm{Cl}=2.474,2.472 ; \mathrm{Ru}- \\
\mathrm{P}=2.314,2.324\end{array}$ & 167,105 & $\begin{array}{l}\mathrm{M}-\mathrm{C} \\
\mathrm{C}_{\mathrm{xyl}}{ }^{-}\end{array}$ \\
\hline $7 a$ & $\mathrm{RuCl}_{2}\left(\mathrm{PPh}_{2}\left(2-\mathrm{Me}-\mathrm{C}_{6} \mathrm{H}_{4}\right)_{2}\right.$ & simplified 7 & $\begin{array}{l}\mathrm{Ru}-\mathrm{Cl}=2.401,2.398 ; \mathrm{Ru}- \\
\mathrm{P}=53588 * 2\end{array}$ & 143,99 & $\begin{array}{l}\mathrm{M}-\mathrm{C} \\
\mathrm{C}_{\mathrm{Ph}}-\end{array}$ \\
\hline
\end{tabular}


Table S2: Cont'd

\begin{tabular}{|c|c|c|c|c|c|}
\hline No. & Complex & Input Description & M-L / Å & A1, A2 $/^{\circ}$ & Oth \\
\hline 8 & {$\left[\mathrm{RuPh}(\mathrm{CO})\left(\mathrm{P}^{\mathrm{t}} \mathrm{Bu}_{2} \mathrm{Me}\right)_{2}\right]$} & From XRD & $\begin{array}{l}\mathrm{Ru}-\mathrm{C}(\mathrm{O})=1.819 ; \mathrm{Ru}-\mathrm{P}= \\
2.457,2.454 ; \mathrm{Ru}-\mathrm{C}(\mathrm{Ph})= \\
2.040\end{array}$ & 164,93 & M-C \\
\hline $8 \mathbf{8 a}$ & {$\left[\mathrm{RuPh}(\mathrm{CO})\left(\mathrm{PMe}_{3}\right)_{2}\right]$} & $\begin{array}{l}\text { simplified } 8 \\
\text { Tetrahedral } \\
\text { Square planar }\end{array}$ & $\begin{array}{l}\mathrm{Ru}-\mathrm{C}(\mathrm{O})=1.824 ; \mathrm{Ru}-\mathrm{P}= \\
2.429,2.427 ; \mathrm{Ru}-\mathrm{C}(\mathrm{Ph})= \\
2.028 \\
\mathrm{Ru}-\mathrm{C}(\mathrm{O})=1.824 ; \mathrm{Ru}-\mathrm{P}= \\
2.426,2.429 ; \mathrm{Ru}-\mathrm{C}(\mathrm{Ph})= \\
2.027 \\
\mathrm{Ru}-\mathrm{C}(\mathrm{O})=1.979 ; \mathrm{Ru}-\mathrm{P}= \\
2.498,2.382, \mathrm{Ru}-\mathrm{C}(\mathrm{Ph})= \\
2.022\end{array}$ & $\begin{array}{l}174,97 \\
175,97 \\
178,167\end{array}$ & M-C \\
\hline 11 & $\mathrm{FeCl}_{3}\left(\mathrm{PPh}_{3}\right)_{2}$ & $\begin{array}{l}\text { From XRD, } \mathrm{S}=1 / 2 \\
\text { From } \mathrm{XRD}, \mathrm{S}=3 / 2 \\
\text { From } \mathrm{XRD}, \mathrm{S}=5 / 2\end{array}$ & $\begin{array}{l}\mathrm{Fe}-\mathrm{Cl}=2.209,2.241, \\
2.220 ; \mathrm{Fe}-\mathrm{P}=2.385,2.379 \\
\mathrm{Fe}-\mathrm{Cl}=2.304,2.265, \\
2.299 ; \mathrm{Fe}-\mathrm{P}=2.387,2.393 \\
\mathrm{Fe}-\mathrm{Cl}=2.260,2.262, \\
2.271 ; \mathrm{Fe}-\mathrm{P}=2.666,2.670\end{array}$ & & 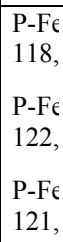 \\
\hline 12 & $\mathrm{FeCl}_{3}\left(\mathrm{PMe}_{3}\right)_{2}$ & $\begin{array}{l}\text { From } \mathrm{XRD}, \mathrm{S}=1 / 2 \\
\text { From } \mathrm{XRD}, \mathrm{S}=3 / 2 \\
\text { From } \mathrm{XRD}, \mathrm{S}=5 / 2\end{array}$ & $\begin{array}{l}\mathrm{Fe}-\mathrm{Cl}=2.225,2.255 \\
2.225 ; \mathrm{Fe}-\mathrm{P}=2.339,2.334 \\
\mathrm{Fe}-\mathrm{Cl}=2.306,2.276, \\
2.306 ; \mathrm{Fe}-\mathrm{P}=2.349,2.348 \\
\mathrm{Fe}-\mathrm{Cl}=2.278,2.278, \\
2.296 ; \mathrm{Fe}-\mathrm{P}=2.586,2.587\end{array}$ & & 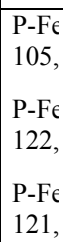 \\
\hline
\end{tabular}

Table S2: Cont'd 


\begin{tabular}{|c|c|c|c|c|c|}
\hline No. & Complex & Input Description & M-L / Å & $\mathbf{A 1}, \mathbf{A} 2 /^{\circ}$ & Oth \\
\hline 14 & {$\left[\mathrm{Ru}(\mathrm{tmbp})_{2}\right]$} & $\begin{array}{l}\text { From XRD, no } \\
\text { counterion } \\
\text { Tetrahedral }\end{array}$ & $\begin{array}{l}(2.260,2.261) * 2 \\
2.245 .2 .248 * 2,2.251\end{array}$ & $\begin{array}{l}154,154 \\
128,127\end{array}$ & $\begin{array}{l}\mathrm{tmb} \\
\mathrm{tmb}\end{array}$ \\
\hline 14a & {$\left[\mathrm{Ru}(\mathrm{bp})_{2}\right]^{2-}$} & $\begin{array}{l}{\left[\mathrm{Ru}(\mathrm{bp})_{2}\right]^{2-} \text {, removed }} \\
\text { Me groups from tmbp } \\
\text { ligands, simplified } \\
\text { XRD } \\
\text { Tetrahedral } \\
\text { Square planar, } D_{2 h} \\
\text { Broken symmetry } \\
\text { Broken symmetry, } D_{2 h}\end{array}$ & $\begin{array}{l}(2.253,2.254) * 2 \\
2.244,2.246,2.247,2.250 \\
2.267 * 4 \\
(2.242,2.361) * 2 \\
2.364 * 4\end{array}$ & $\begin{array}{l}148,148 \\
129,127 \\
180,180 \\
172,172 \\
180,180\end{array}$ & $\begin{array}{l}\text { tmb] } \\
\text { tmb] } \\
\text { tmb] } \\
\text { tmb] } \\
\text { plan } \\
\text { tmb }\end{array}$ \\
\hline $\begin{array}{l}14 \\
b\end{array}$ & {$\left[\mathrm{Ru}(\mathrm{tmbp})_{2}\right]\left[\mathrm{Li}(\mathrm{thf})_{3}\right]$} & From XRD & $(2.259,2.265) * 2$ & 167,166 & $\begin{array}{l}\mathrm{tmb}] \\
2.75\end{array}$ \\
\hline 15 & $\mathrm{PdCl}_{2}\left(\mathrm{PMe}_{2} \mathrm{Ph}\right)_{3}$ & From XRD & $\begin{array}{l}\mathrm{Pd}-\mathrm{Cl}=2.703,2.559 ; \mathrm{Pd}- \\
\mathrm{P}=2.357,2.358,2.375\end{array}$ & & $\begin{array}{l}\mathrm{Cl}-\mathrm{F} \\
\mathrm{P}= \\
\circ\end{array}$ \\
\hline $15 a$ & {$\left[\mathrm{PdCl}\left(\mathrm{PMe}_{2} \mathrm{Ph}\right)_{3}\right]^{+}$} & $\begin{array}{l}\text { Removed axial } \mathrm{Cl} \\
\text { from XRD }\end{array}$ & $\begin{array}{l}\mathrm{Pd}-\mathrm{Cl}=2.378 ; \mathrm{Pd}-\mathrm{P}= \\
2.399,2.433,2.363\end{array}$ & 178,170 & $\begin{array}{l}\text { Max } \\
\text { Pd-I }\end{array}$ \\
\hline $\begin{array}{l}15 \\
\text { b }\end{array}$ & $\begin{array}{l}{\left[\mathrm{PdCl}_{2}\left(\mathrm{PMe}_{2} \mathrm{Ph}\right)_{3}\right] \cdot \mathrm{CH}_{2} \mathrm{Cl}_{2} \quad \text { (close }} \\
\text { contact in crystal structure) }\end{array}$ & From XRD & $\begin{array}{l}\mathrm{Pd}-\mathrm{Cl}=2.796,2.508 ; \mathrm{Pd}- \\
\mathrm{P}=2.368,2.364,2.353\end{array}$ & & $\begin{array}{l}\text { Cl-F } \\
\mathrm{P}= \\
\mathrm{P}=\end{array}$ \\
\hline
\end{tabular}


Table S2: Cont'd

\begin{tabular}{|c|c|c|c|c|c|}
\hline No. & Complex & Input Description & M-L / Å & $\mathrm{A} 1, \mathrm{~A} 2 /^{\circ}$ & Oth \\
\hline $15_{\text {aq }}$ & $\mathrm{PdCl}_{2}\left(\mathrm{PMe}_{2} \mathrm{Ph}\right)_{3}$ & $\begin{array}{l}\text { in water continuum } \\
\text { dielectric field }\end{array}$ & $\begin{array}{l}\mathrm{Pd}-\mathrm{Cl}=2.970,2.511 ; \mathrm{Pd}-\mathrm{P} \\
=2.392,2.392,2.365\end{array}$ & & $\begin{array}{l}\mathrm{Cl}-\mathrm{I} \\
\mathrm{P}= \\
\mathrm{\circ}\end{array}$ \\
\hline 18a & {$\left[\mathrm{Cr}(\mathrm{en})_{2} \mathrm{Ox}\right]^{+}$} & From XRD & $\begin{array}{l}\mathrm{Cr}-\mathrm{N}=2.144,2.145 \\
2.196 * 2 ; \mathrm{Cr}-\mathrm{O}=1.885 * 2\end{array}$ & & \\
\hline 20 & {$\left[\mathrm{Co}(\mathrm{en})_{2} \mathrm{Ox}\right]^{+}$} & Modified 18a & $\begin{array}{l}\mathrm{Co}-\mathrm{N}=1.987,1.989 \\
2.033,2.036 ; \mathrm{Co}-\mathrm{O}= \\
1.859,1.860\end{array}$ & & \\
\hline 21 & {$\left[\mathrm{PdCl}_{2}\left(\mathrm{dppfO}_{2}\right)\right]$} & $\begin{array}{l}\text { From XRD, } \mathrm{S}=0 \\
\text { From } \mathrm{XRD}, \mathrm{S}=1\end{array}$ & $\begin{array}{l}\mathrm{Pd}-\mathrm{Cl}=2.311,2.310 ; \text { Pd- } \\
\mathrm{O}=2.133,2.136 \\
\mathrm{Pd}-\mathrm{Cl}=2.427,2.369 ; \text { Pd- } \\
\mathrm{O}=2.206,2.281\end{array}$ & $\begin{array}{l}177,177 \\
145,94\end{array}$ & \\
\hline 22 & {$\left[\mathrm{NiCl}_{2}\left(\mathrm{dppfO}_{2}\right)\right]$} & $\begin{array}{l}{\left[\mathrm{NiCl}_{2}\left(\mathrm{dppfO}_{2}\right)\right], \mathrm{S}=0} \\
\mathrm{~S}=1\end{array}$ & $\begin{array}{l}\mathrm{Ni}-\mathrm{Cl}=2.198,2.205 ; \mathrm{Ni}- \\
\mathrm{O}=1.945,1.960 \\
\mathrm{Ni}-\mathrm{Cl}=2.256,2.289 ; \mathrm{Ni}- \\
\mathrm{O}=1.996,2.008\end{array}$ & $\begin{array}{l}177,175 \\
137,100\end{array}$ & \\
\hline 23 & cis- $\left[\mathrm{RuCl}_{2}\left(\mathrm{C} \equiv \mathrm{N}-\left(2,6-\mathrm{Me}_{2}-\mathrm{Ph}\right)\right)_{2}\right]$ & $\begin{array}{l}\text { From XRD, } \mathrm{S}=0 \\
\text { From } \mathrm{XRD}, \mathrm{S}=1\end{array}$ & $\begin{array}{l}\mathrm{Ru}-\mathrm{Cl}=2.363,2.364 ; \mathrm{Ru}- \\
\mathrm{C}=1.869,1.869 \\
\mathrm{Ru}-\mathrm{Cl}=2.370,2.374 ; \mathrm{Ru}- \\
\mathrm{C}=1.991,1.998\end{array}$ & $\begin{array}{l}156,96 \\
168,165\end{array}$ & \\
\hline 24 & cis-[PdCl$\left.\left(\mathrm{C} \equiv \mathrm{N}-\left(2,6-\mathrm{Me}_{2}-\mathrm{Ph}\right)\right)_{2}\right]$ & From $\mathrm{XRD}, \mathrm{S}=0$ & $\begin{array}{l}\mathrm{Ru}-\mathrm{Cl}=2.352,2.352 ; \mathrm{Ru}- \\
\mathrm{C}=1.964,1.964\end{array}$ & 177,177 & \\
\hline
\end{tabular}




\section{References}

1. Kersten, J. L.; Kucharczyk, R. R.; Yap, G. P. A.; Rheingold, A. L.; Theopold, K. H., $\left[\left(\mathrm{Tp}^{\mathrm{tBu}, \mathrm{Me}}\right) \mathrm{CrR}\right]:$ An New Class of Mononuclear, Coordinatively Unsaturated Chromium(II) Alkyls with cis-Divacant Octahedral Structure. Chem. Eur. J. 1997, 3, 1668-1674.

2. MacAdams, L. A.; Kim, W.-K.; Liable-Sands, L. M.; Guzei, I. A.; Rheingold, A. L.; Theopold, K. H., The $(\mathrm{Ph})_{2}$ nacnac Ligand in Organochromium Chemistry. Organometallics 2002, 21, 952-960.

3. Ogasawara, M.; Macgregor, S. A.; Streib, W. E.; Folting, K.; Eisenstein, O.; Caulton, K. G., Characterzation and Reactivity of an Unprecedented Unsaturated Zero-Valent Ruthenium Species: Isolable, Yet Highly Reactive. J. Am. Chem. Soc. 1996, 118, 10189-10199.

4. Bröring, M.; Prikhodovski, S.; Brandt, C. D., The first (tripyrrinato)nickel(II) complexes, TrpyNiX with X-Cl, Br, I: synthesis, structure and solvent coordination. J. Chem. Soc., Dalton Trans. 2002, $4213-4218$.

5. Watson, L. A.; Ozerov, O. V.; Pink, M.; Caulton, K. G., Four-Coordinate, Planar Ru(II). A Triplet State as a Response to a 14-Valence Electron Configuration. J. Am. Chem. Soc. 2003, 125, 8426-8427. 